\title{
Introduction: Integrated Approaches to Studying the Development of Emotion
}

\author{
Kristin Hansen Lagattuta \\ Department of Psychology and Center for Mind and Brain, University of California, Davis, Calif., USA
}

Emotions are central to everyday human lives and relationships. How we attend to, interpret, talk about and respond to emotional displays of others, regulate our own internal feelings, reminisce about emotions from the past, and anticipate our future affective reactions critically influences our well-being and decision making, including how we form, maintain, and confront challenges in social relationships. For these reasons, developmental scientists have focused strong research attention on the development of emotion processing, understanding, and regulation in infancy and childhood, including sources of individual differences (e.g. genetics, biology, family, culture, early experience). A basic search in July 2013 on the PsycINFO behavioral sciences database for 'children and emotion' yielded 18,649 entries, 'development and emotion' 22,971, and 'emotional development' 53,280 - with all categories showing rapid growth in publication numbers over the past 10 years. Indeed, for all three searches, more than $50 \%$ of the total scholarly works were published within the past decade alone.

The chapters in this volume summarize and critically evaluate cutting-edge research on children and emotion. In selecting topics and authors for this book, I aimed to incorporate leaders and rising stars in the field who, as a group, use multiple levels of analysis (behavioral, cognitive, so- cial, neural, genetic) and diverse research methods (e.g. observational studies, experimental studies, narrative analyses, self-report measures, parent-report measures, eye-tracking, heart rate, cortisol, ERP, fMRI). Together, the nine chapters cover age-related changes and individual variability in infants' and children's attention, processing, understanding, conversation, regulation, and expression of emotions in typical and atypical populations, including connections to parenting and wider cultural values and norms. Due to the centrality of emotions to children's (and adults') lives and experiences, this book will be useful to researchers, educators, parents, and policy makers. Below, I highlight the key features of each contribution.

In the first chapter, Hoehl critically reviews behavioral (preferential looking, habituation, eye-tracking) and neurophysiological research (ERP studies) on how infants attend to, process, and discriminate facial emotion expressions during the first year of life. Of central focus is the development of attentional biases towards negative emotions (especially anger and fear), including variations in attention (e.g., to negative vs. positive emotion faces, to eye vs. mouth regions) based on whether the person expressing the emotion is looking directly ahead or at a specific referent. She further explores how variability in infant 
temperament, genetics, parenting, and experience shape how infants process and respond to emotion signals. Hoehl ends by urging researchers to integrate multiple technologies and levels of analysis (e.g., eye tracking, neurophysiological measures, behavior) to advance the science of infant emotion processing.

The second chapter, by Hastings, Kahle and Han, explores the benefits and complexities of incorporating assessments of biological activity into developmental emotion research. Developmental scientists are more frequently relying on psychophysiological techniques (e.g., neurophysiological, neuroendocrinological, autonomic, electromyographic measures) to understand how children regulate their emotions, as well as how children use emotion to regulate cognition and behavior. As Hastings and colleagues aptly argue, this research enterprise poses a significant challenge because 'physiological systems are developing, emotions are developing, and the relations between physiology and emotions also are developing'. As with Hoehl's chapter, Hastings and colleagues further discuss sources of individual differences in relation to temperament, genetics, parenting, and early experience, and they provide ideas for future work.

Examination of atypical life experiences and maladaptive developmental patterns can help elucidate the mechanisms of normal development. Thus, in the third chapter, Cicchetti and Ng investigate the impact of physical maltreatment on how young children perceive, regulate, process, express, and understand emotions. The authors argue that deviations in all of these areas begin during infancy, with these perturbations both reflecting and strengthening the abnormal development of emotion-related neural networks. Converging evidence from behavioral and neuroscience approaches support these interpretations, especially regarding relations between abusive home environments and the development of hypervigilance to anger. In addition to giving multiple suggestions for future research,
Cicchetti and Ng further address how scientific inquiry can inform the design of effective interventions.

Pérez-Edgar, Taber-Thomas, Auday and Morales continue this discussion of atypical emotional development in their chapter by exploring the emergence of childhood anxiety. They argue that although temperament is a significant risk factor for developing anxiety (especially behavioral inhibition or temperamental shyness), children's attention biases to threat-related information modulate this link between temperament and anxiety. Pérez-Edgar and colleagues draw from behavioral (e.g., dot-probe tasks), psychophysiological (e.g., eye tracking) and neuroscience research (ERP, fMRI) to substantiate this temperament-attention-anxiety network. They further describe how training programs aimed at modifying attention biases provide a promising approach for both preventing and reducing anxiety symptoms in children and adults.

In the fifth chapter, Lemerise and Harper shift emphasis towards how children develop emotional competence in the context of family and peer relationships. Multiple studies converge to indicate that warm and supportive parent-child relationships provide an essential foundation for children to develop skills at identifying, understanding, regulating, talking about, and responding to their own and others' emotions. Moreover, individual differences in emotional competence predict children's concurrent and future peer relations and behavioral adjustment. The authors close with arguing that intervention programs designed to improve preschoolers' emotion knowledge and emotion regulatory skills can help reduce socio-emotional problems and academic difficulties in the critical transition to school.

Because emotion researchers have concentrated primarily on Western populations, the literature provides an imbalanced perspective on emotional development. Thus, in the chapter by Camras, Shuster, and Fraumeni, they take a crosscultural approach when considering relations be- 
tween parents' beliefs, attitudes, and values, their parenting practices, and children's emotional competence. They use numerous research examples to highlight how the same emotions can be interpreted, valued, or expressed in different ways in different cultures. Moreover, the same parenting behaviors can be motivated by different beliefs about emotions and predict different child outcomes. The authors end with strategies for building research collaborations with non-Western nations; partnerships essential for constructing comprehensive theories of emotion.

Next, Fivush delves deeper into one of the central ways in which children learn about emotions - through parentally guided conversations about past emotional experiences. Using sociocultural and feminist theories to frame her arguments, Fivush proposes that through parent-child reminiscing about the past children develop an autobiographical voice that reflects their understanding of their own and others' emotional lives. Cross-sectional and longitudinal studies in childhood and adolescence reveal significant differences in how mothers versus fathers discuss emotions as well as how parents talk about emotions with sons versus daughters. In turn, these co-constructed narratives shape how children learn to interpret, express, and regulate emotions appropriate for their social, historical, and cultural place.

The next chapter expands this focus on family conversation to investigate how parent-child conversations about internal mental states more broadly - about emotions, thoughts, and desires - not only relate to children's growing emotion knowledge but also to their understanding of their own and others' psychological lives (theory of mind). Hughes, White and Ensor critically review a broad base of literature demonstrating how children's linguistic environments, especially parent-child talk about emotions and the mind, causally shape their social-cognitive development. They consider not only typically developing children in Western samples, but also children with deafness, children diagnosed with au- tism, and children from non-Western cultural communities. Training and intervention studies help to elucidate further the mechanisms by which parent-child conversations impact children's learning about the social world.

In the final chapter, Harris, de Rosnay and Ronfard provide a thoughtful analysis of an intriguing paradoxical pattern in young children's understanding of emotions - a lag between their understanding that a person can have a mistaken (or false) belief and their ability to infer the person's emotions based on this belief. For example, whereas 4- to 6-year-olds frequently state that Little Red Riding Hood thinks her grandmother is lying in the bed (as opposed to a disguised, threatening wolf) they nevertheless predict that she will feel afraid (because it really is the wolf). That is, when children forecast emotions, the reality of the situation often trumps what they know about belief. The authors evaluate several alternative explanations for this disconnect between children's belief and emotion judgments, and they suggest avenues for future research on developmental changes and individual differences in children's emotion understanding.

This book project would not have taken off without the enthusiastic participation of this elite group of researchers. I am extremely grateful for their informative, thought-provoking, and research-inspiring chapters. I greatly admire their methodological rigor and creative approaches to examining emotion from the lens of development. I also wish to thank Larry Nucci, the editor of this volume series, for giving me this opportunity, the editorial staff at Karger for their assistance with the production and promotion of the book (especially Sandra Braun, Brigitte Thierstein, and Angela Gasser), my graduate and undergraduate students who helped in the editing process (especially Hannah Kramer), and my husband and three children for providing continued support and multiple opportunities to learn first-hand about emotion in the lives of children and families. 